\title{
Técnicas de la PNL utilizadas por los líderes de las empresas mixtas del sector petrolero
}

\author{
NLP techniques used by leaders of joint ventures in the oil sector
}

Técnicas de PNL usadas por líderes de joint ventures no setor de petróleo

Recibido: enero 2021

Arbitrado: febrero 2021

Aceptado: marzo 2021

Publicado: mayo 2021

\author{
Armando José Bozo Luzardo \\ armandojbozol@gmail.com \\ https://orcid.org/0000-0002-1241-5196 \\ Universidad Rafael Belloso Chacín, Zulia-Venezuela
}

RESUMEN

La investigación tuvo como objetivo describir las técnicas de la PNL utilizadas por los líderes de las empresas mixtas del sector petrolero. La metodología fue del tipo descriptiva, con un diseño de campo, transaccional. La población estuvo constituida por veinticinco líderes de proyectos de las empresas mixtas del sector petrolero, la técnica e instrumento utilizado fue un cuestionario, constituido por cincuenta y dos ítems, el cual fue validado por cinco expertos del área. Los resultados indicaron que, los líderes si hacen uso de las técnicas del PNL, por lo que se concluye que, pudieran obtener un mayor beneficio sí logran mayor conocimiento y manejo de las técnicas PNL, especialmente la técnica del reencuadre, por lo tanto, estas herramientas son relevantes para el logro de un proceso de liderazgo efectivo en los líderes de las empresas petroleras.

Palabras clave: Programación neurolingüística; gestión de proyectos; tipos de PNL, líderes
The research aimed to describe the NLP techniques used by the leaders of joint ventures in the oil sector. The methodology was descriptive, with a field, transactional design. The population consisted of twenty-five project leaders from joint ventures in the oil sector. The technique and instrument used was a questionnaire, consisting of fifty-two items, which was validated by five experts in the area. The results indicated that leaders do use NLP techniques, which is why it is concluded that they can obtain a greater benefit if they achieve greater knowledge and management of NLP techniques, especially the reframing technique, therefore, tools They are relevant for the achievement of an effective leadership process in the leaders of the oil companies.

Palabras clave: Neuro-linguistic programming; Projects management; types of NLP, leaders

\section{RESUMO}

A pesquisa teve como objetivo descrever as técnicas de PNL utilizadas pelos líderes de joint ventures no setor de petróleo. A metodologia foi descritiva, com desenho transacional de campo. A população foi constituída por vinte e cinco líderes de projetos de joint ventures do setor petrolífero. A técnica e o instrumento utilizados foi um questionário, composto por cinquenta e dois itens, validado por cinco especialistas na área. Os resultados indicaram que os líderes utilizam técnicas de PNL, portanto, conclui-se que eles poderiam obter um maior benefício se obtivessem maior conhecimento e gerenciamento das técnicas de PNL, principalmente a técnica de ressignificação, portanto, essas Ferramentas são relevantes para o alcance de uma liderança processo nos dirigentes das empresas petrolíferas.

Palabras clave: Programação neurolinguística; gestão de projetos; tipos de PNL, líderes 


\section{INTRODUCCIÓN}

L a industria petrolera venezolana comparte muchas de las características que definen a la industria petrolera latinoamericana, incluyendo a la que está emergiendo, pero se diferencia también en muchos aspectos, debido a que constituye un caso "sui géneris" en América Latina. Según las ideas planteadas por García (2004), Venezuela posee una industria petrolera integrada, aleatoria, intensiva en capital, de carácter internacional y es considerada todavía, aunque cada vez menos, una industria básica.

Ciertamente, se sabe que los países latinoamericanos productores de petróleo, al igual en el Estado venezolano son accionistas únicos de grandes corporaciones petroleras. Para el caso de Venezuela, Petróleos de Venezuela, Sociedad Anónima (PDVSA), es considerada en tiempos recientes y según determinados indicadores de mercado, financiero, producción, entre otros, como una de las grandes corporaciones petroleras mundiales; no obstante, la presencia de las empresas mixtas aún se hace notoria con las nuevas alianzas estratégicas de inversión privada en la nación.

En opinión de Gordillo (2005), "el nombre de sociedades de economía mixta puede aplicarse principalmente a aquellas empresas bajo un régimen especial en coparticipación accionaria de capitales del Estado y privados. En estas la participación estatal es estatutaria, así como permanente" (p. 403). Las empresas mixtas son aquellas que reciben aportes de capital de particulares y del estado, es decir, un aporte representado en capital social donde intervienen personas jurídicas de capital privado, y por otro lado, el estado como persona de derecho público.

Por consiguiente, las empresas mixtas en el sector petrolero venezolano, lo primero que han de tener en consideración es la unilateralidad asociada a este hecho, donde el estado venezolano sea socio mayoritario de los resultados de la producción, o expresado en otras palabras, el rompimiento de una mutualidad producida después de la constitución de estas empresas en el año 2006. Asimismo, según la afirmación de Gordillo (2005), el elemento definitorio de la empresa mixta radica en la participación del estado aportando capital, junto al privado, lo cual resulta beneficioso para el sector petrolero, puesto que se acuerdan inversiones, se obtienen financiamientos a nuevos proyectos de exploración y perforación en la industria venezolana actual.

Por otra parte, las empresas mixtas desarrollan este tipo de inversiones a través de proyectos, por lo que, es importante una adecuada gestión, sin embargo, la misma ha pasado por cambios en las dos últimas décadas. Por un tiempo, los proyectos eran administrados de forma "ad hoc", es decir, para estos era designado un gerente con experiencia técnica previa en aquel determinado asunto, no obstante, los índices de fallas llevaron a cambios progresivos en la forma de dirigirlos. Por ende, surge una nueva gestión que aplica métodos, técnicas y mejores prácticas en diferentes complejidades, con un enfoque gerencial no sólo técnico por parte de la figura del líder.

En tal sentido, es relevante destacar que la gestión de proyectos es una disciplina de planificación, la organización, la dirección, y el control de los recursos con el propósito de alcanzar uno o varios objetivos. Su aplicabilidad se ha incrementado en las últimas 
décadas, al grado que esta área hoy en día, ofrece una visión más global del deber ser de la administración en el mundo contemporáneo.

Por tanto, vivir en un mundo globalizado genera cambios en las estrategias y planes de comunicación utilizados y desarrollados. Hoy como en el pasado remoto, la comunicación ha sido la manera como los seres humanos han intercambiado emociones, sensaciones y pensamientos para entenderse mutuamente, lo cual ha servido para resolver problemas de manera más rápida y eficaz; el sistema de comunicación debe ser el adecuado, por tanto, las diversas herramientas que los líderes pueden aplicar permiten desempeñar una buena gestión de proyectos, cuyo propósito se centra en gerenciar apropiadamente los mismos, entre ellas la más reconocida es la llamada Programación Neurolingüística (PNL).

Según Sambrano (2005), la programación neurolingüística (PNL) se le denomina "programación" porque trata de un conjunto sistemático de operaciones que persiguen un objetivo; "neuro" porque estudia los procesos ocurridos en el sistema nervioso, y “lingüística” porque para ello es usado el lenguaje, expresado en forma verbal, corporal, entre otros, para organizar la conducta y el pensamiento, por tanto, lograr así una comunicación eficiente con los demás y consigo mismo.

Por las consideraciones anteriores, la comunicación supone una parte importante de en el día a día y se le debería prestar la atención que se merece: un líder de proyectos invierte la mayor parte de su tiempo en comunicarse con su equipo de trabajo, los clientes, los usuarios, los proveedores, los gestores de su organización, compañeros de trabajo con quien interactúa y los interesados del proyecto. Por tanto, su comunicación se realiza de diversas formas: negociando, reportando el estado de un proyecto, asignando trabajo al equipo, obteniendo información de la ejecución del mismo, entre otros. La PNL para los líderes se constituye en una herramienta que permite alcanzar mejoras en el desempeño y en la toma de decisiones.

De acuerdo a García (2004), las empresas venezolanas en los últimos tiempos han determinado que los conflictos personales influyen en la vida laboral y viceversa; por tanto, se necesitan personas con buen estado integral en las organizaciones, especialmente aquellas que tienen la responsabilidad de tomar decisiones; el uso de la herramienta PNL permite mejorar relaciones interpersonales dentro y fuera de la organización, con los clientes, compañeros de trabajo, incrementar la negociación exitosa con los proveedores y generar un clima de confianza.

Tal es el caso de los líderes de proyectos que conforman las empresas mixtas del sector petrolero del estado Zulia, quienes mantienen contacto permanente con los involucrados, pero se puede evidenciar que entre ellos existen constantes malos entendidos debido a las distintas formas de expresar las ideas y opiniones, directrices dadas de forma ambigua, descontrol en la utilización de los recursos asignados, tareas manejadas de manera errónea, e incluso discusiones por puntos de vista diferentes entre el personal que las conforma; inconvenientes que son percibidos por todos los miembros del equipo de trabajo.

Con base a todo lo antes descrito, la investigación tiene como objetivo, describir las técnicas de la PNL utilizadas por los líderes de las empresas mixtas del sector petrolero del estado Zulia. 


\section{Programación Neurolingüística (PNL)}

Según Astorga (2013), la Programación Neurolingüística (PNL) cuenta con una serie de técnicas tales como: el rapport, la calibración, el anclaje, entre otras, las cuales permiten a las personas desarrollar capacidades beneficiosas para el área de salud, gerencial, empresarial, personal, entre otros. Asimismo, Garay (2012), se refiere a esas técnicas como aquellas que permiten desarrollar procesos de comunicación y cambio con excelencia, promoviendo flexibilidad del comportamiento, el pensamiento estratégico, así como la comprensión de los procesos mentales. Pueden ser aplicadas tanto a situaciones personales, grupales y organizacionales basadas en interacciones humanas.

Además, según un artículo publicado en el portal Aprender PNL (2013), sus técnicas son útiles para los cambios de estados mentales, permiten cambiar posturas corporales, asimismo, ayudan a vencer barreras, al igual que de resistencias a los cambios. Por otra parte, Bandler y Grinder (2013), exponen que las técnicas de la PNL habilitan a las personas para mejorar el conocimiento de sí mismo, así como de los demás, lo que hace que los mensajes sean más precisos y congruentes, y se obtienen recursos para decir lo que se piensa, lo que se siente, a modo de concretar los objetivos personales y organizacionales.

En este sentido, al analizar las opiniones anteriores se infiere sobre las técnicas de la PNL, como aquellas que empoderan al individuo para cambiar paradigmas, vencer pensamientos que no dejan crecer personalmente ni laboralmente. La aplicación de dichas técnicas logra describir las preferencias, así como los estilos individuales del pensamiento para comunicarse a través de ellas, además, contribuyen a la resolución de problemas y a la toma de decisiones. El aporte que se realice en función de la utilización de estas técnicas por parte de los líderes creará una diferencia en la gestión de las empresas mixtas petroleras, ya que su aplicación tal y como lo indica Bandler y Grinder (2013) busca mejorar fallas comunicacionales y la generación de climas de producción efectivos.

\section{Técnicas de la Programación Neurolingüística}

\section{Calibración}

Según O’Connor y Seymour (2000), calibración es la palabra empleada por la PNL cuyo significado es reconocer cuándo las personas están en estados mentales diferentes. Ésta es una habilidad que todos tenemos, la cual empleamos en nuestra vida diaria, es muy útil para desarrollar o pulir. Del mismo modo, Sambrano (2005), hace referencia a calibración como la técnica de aprender a conocer el estado mental de las personas, es una habilidad que todos poseemos y empleamos a diario, cuando se desarrolla la habilidad de calibrar, se optimiza o se logra la excelencia en la comunicación.

En este mismo sentido, Carrión (2005), sostiene que la calibración es la observación de una serie de manifestaciones en una persona cuando se evoca una situación sea agradable o no, esto permite obtener una fotografía que permite reconocer cuando está en un estado positivo o por el contrario en uno negativo. Asimismo, para Arocha (2005), la calibración consiste en observar al interlocutor y recoger información externa sobre su fisiología, la información del lenguaje utilizado por el mismo se recoge a través de preguntas. 
De lo anterior, se deriva que es posible ir más allá de la mera intuición para llegar a definir el estado de ánimo del interlocutor, además de posicionarse con la finalidad de calibrar todo comportamiento lo cual supone una actividad neurológica determinada por los sentimientos, las experiencias pasadas, las sensaciones internas, los pensamientos. Según lo establecido por Sambrano (2005), observar a las personas, escuchar su discurso, fijarse en sus gestos y posturas, son señales que pueden ayudar a calibrar su estado interno, al descubrir los indicadores comportamentales asociados.

Es por lo anteriormente planteado que, calibrar a las personas y en el caso de esta investigación, a los involucrados en los proyectos, permite al mismo tiempo conocer el sistema de representación predominante en cada individuo para establecer una comunicación congruente con su sistema de representación lo cual aumenta la posibilidad de que esta sea efectiva.

\section{Anclaje}

En este mismo orden de ideas, O’Connor y Seymour (2000), expresan que el anclaje está referido a los estímulos asociados a estados psicológicos, tales como: ver sus fotografías favoritas, olores evocadores, una expresión especial de alguien querido o un tono de voz. Por su parte, Mohl (2006), manifiesta que establecer un estímulo externo y ligarlo deliberadamente con la experiencia, con el objeto de resucitarla en el momento deseado, en términos de PNL se le denomina, anclaje.

Asimismo, Carpio y Flores (2007), definen al anclaje como un proceso de condicionamiento emocional en el cual una situación, experiencia o estado emocional del pasado del individuo es asociado a un estímulo específico y reaparece en el presente con determinadas consecuencias. Asimismo, Sambrano (2005), concibe un ancla, como aquella palabra, gesto, olor, color, gusto que nos lleva a un estado mental determinado porque se estableció así de alguna forma en el pasado.

De acuerdo al planteamiento de Mohl (2006), el proceso de anclaje consiste en llevar a las personas a un estado anímico que involucre, recuerdos, sensaciones, emociones o pensamientos que sirvan para manifestar opiniones, así como gratitudes ligadas a las experiencias o vivencias del ser humano.

Lo anteriormente expuesto evidencia que las teorías de varios autores convergen al conceptualizar una situación muchas veces vivida por los seres humanos, tal técnica sería de utilidad para los líderes de proyectos al momento de cambiar la opinión de sus colaboradores o equipos de trabajo, en tales casos como; una reunión aclaratoria con la contratista, una reunión de avance del proyecto, discusión de controles de calidad de los entregables, reconocimiento a sus involucrados, integración de los interesados en el proyecto, entre otros importantes.

\section{Rapport}

Según lo expuesto por Mohl (2006), la sintonía o (rapport) es la existencia de un contacto directo entre las personas, también la define como la relación entre dos individuos, caracterizada por el respeto y la confianza mutua. La calidad de la sintonía dependerá 
básicamente de la actitud interna del individuo, de su capacidad para aceptar al otro, pero también de su disposición momentánea: es necesario que se sienta receptivo para dedicarse plenamente a su interlocutor.

En opinión de Sambrano (2005), rapport significa armonía, concordancia, simpatía; es la habilidad para colocarse en el lugar del otro, "sintonizar" con los sentimientos para enriquecer el carácter de las relaciones que se establecen con los semejantes y mejorar así, la calidad del proceso de la comunicación. Según Carrión (2005), se define el rapport como la penetración, así como la adaptación del propio mapa o modelo del mundo, al mapa de otra u otras personas.

De acuerdo a lo manifestado por los autores, las personas establecen rapport colocándose en la posición de otros, cuando analizan mapas o modelos de percepción del mundo, lo cual según Mohl (2006), crea empatía entre las personas, sintonizando actitudes en los demás, en la forma de pensar de los seres humanos, lo que hace al individuo más receptivo a las diversas corrientes del pensamiento; por consiguiente, si se crea una sinergia entre los líderes de proyectos así como con sus colaboradores, se abrirá un mundo de posibilidades de receptividad al cambio en los procesos de trabajo, lo que trae consigo, asignación de responsabilidades de manera más asertiva en los proyectos, y se promoverá la proactividad y la buena toma de decisiones en los involucrados en la ejecución de los proyectos.

\section{Reencuadre}

El reencuadre para los líderes de proyectos, es una técnica provechosa por cuanto se refiere a replantear una idea o contextualizar de otra manera una situación característica para adaptarla a una situación positiva. En palabras de Sambrano (2005), consiste en modificar el marco de referencia en el cual una persona percibe los hechos para cambiar su significado, cuando este varía, también cambia el estado emocional, las respuestas y las conductas de las personas. Asimismo, O' Connor y Seymour (2000), infieren que el significado de cualquier evento, cambia cuando se cambia el marco, por lo que, al cambiar el significado, también varían las respuestas y el comportamiento.

Por otro lado, Carpio y Perera (2009), definen el reencuadre bajo los supuestos: la gente tiene los recursos que necesita para cambiar, ninguna conducta es mala o negativa en sí misma. En consideración a lo expuesto por los anteriores autores, se entiende como reencuadre aquel hecho donde no se ve afectada la intención de lo dicho o la actuación, sino que prepondera su significado y en el cual de acuerdo a lo manifestado por O' Connor y Seymour (2000), la acción de reencuadrar se enfatiza en el objeto de cambiar algún significado o apreciación, con la finalidad de reestructurar la conducta de las personas en la organización para obtener luego las mejores respuestas.

En tal consideración, obtener el cambio de marco de referencia en los líderes o colaboradores de proyectos puede beneficiar las estrategias para el desarrollo de los mismos, puesto que permite crear un ambiente motivador en reuniones de negocios, con clientes, proveedores, al tratar de persuadir al interlocutor y lograr el involucramiento necesario con una realidad o percepción de alguna idea de proyecto, por otra parte, concede entre sus bondades una técnica para la resolución de conflictos internos. 


\section{MÉTODO}

$\mathrm{I}$ a investigación fue de tipo descriptiva, con un diseño de campo, transaccional. La población estuvo conformada por veinticinco líderes de proyectos, el instrumento aplicado para la recolección de la información fue un cuestionario, constituido por cincuenta y dos (52) ítems, el cual fue validado por cinco expertos del área y se midió su confiabilidad a través del indicador Alfa de Cronbach el cual arrojó un valor de 0,972 que lo ubica en la categoría muy alta. Los datos obtenidos fueron tabulados e interpretados mediante la estadística descriptiva, basándose en las medias de las dimensiones e indicadores. Para el análisis estadístico de los resultados se diseñó un baremo de interpretación, el cual se muestra a continuación en la Tabla 1.

Tabla 1. Baremo de interpretación de los promedios. Variables: Programación neurolingüística y Gestión de proyectos.

\begin{tabular}{|c|c|}
\hline RANGO & INTERPRETACIÓN \\
\hline $1-1,80$ & Muy Baja Aplicación \\
\hline $1,81-2,60$ & Baja Aplicación \\
\hline $2,61-3,40$ & Moderada Aplicación \\
\hline $3,41-4,20$ & Alta aplicación \\
\hline $4,21-5$ & Muy Alta Aplicación \\
\hline
\end{tabular}

\section{RESULTADOS Y DISCUSIÓN}

L a Tabla 2 muestra los resultados obtenidos por la población encuestada sobre las características de las técnicas de la PNL utilizadas por los líderes de las empresas mixtas del sector petrolero del estado Zulia.

Tabla 2. Distribución frecuencial de la dimensión técnicas de la PNL utilizadas por líderes de empresas.

\begin{tabular}{|c|c|c|c|c|c|c|c|c|c|c|}
\hline \multirow{2}{*}{\multicolumn{2}{|c|}{$\begin{array}{l}\text { Indicadores } \\
\text { Ítems }\end{array}$}} & \multicolumn{2}{|c|}{ Calibración } & \multicolumn{3}{|c|}{ Anclaje } & \multicolumn{2}{|c|}{ Rapport } & \multicolumn{2}{|c|}{ Reencuadre } \\
\hline & & \multirow{2}{*}{$\begin{array}{c}1314 \\
\text { FA }\end{array}$} & \multirow{2}{*}{$\begin{array}{c}1516 \\
\text { FR \% }\end{array}$} & \multirow{2}{*}{$\begin{array}{l}17 \\
\text { FA }\end{array}$} & \multirow{2}{*}{18} & \multirow{2}{*}{$\begin{array}{c}1920 \\
\text { FR \% }\end{array}$} & \multirow{2}{*}{$\begin{array}{c}2122 \\
\text { FA }\end{array}$} & \multirow{2}{*}{$\begin{array}{c}23 \quad 24 \\
\text { FR \% }\end{array}$} & \multirow{2}{*}{$\begin{array}{c}2526 \\
\text { FA }\end{array}$} & \multirow{2}{*}{$\begin{array}{c}27 \quad 28 \\
\text { FR \% }\end{array}$} \\
\hline Alternativas & Valor & & & & & & & & & \\
\hline Siempre & 5 & 9 & 36,0 & 9 & & 36,0 & 11 & 44,0 & 7 & 28,0 \\
\hline Casi siempre & 4 & 4 & 16,0 & 7 & & 28,0 & 7 & 28,0 & 6 & 24,0 \\
\hline Algunas veces & 3 & 7 & 28,0 & 3 & & 12,0 & 4 & 16,0 & 5 & 20,0 \\
\hline Casi nunca & 2 & 3 & 12,0 & 5 & & 20,0 & 1 & 4,0 & 5 & 20,0 \\
\hline Nunca & 1 & 2 & 8,0 & 1 & & 4,0 & 2 & 8,0 & 2 & 8,0 \\
\hline Total & & 25 & 100 & 25 & & 100 & 25 & 100 & 25 & 100 \\
\hline \multicolumn{2}{|c|}{ Media del indicador } & \multicolumn{2}{|c|}{3,53} & \multicolumn{3}{|c|}{3,50} & \multicolumn{2}{|c|}{3,75} & \multicolumn{2}{|c|}{3,37} \\
\hline \multicolumn{2}{|c|}{ Categoría del indicador } & \multicolumn{2}{|c|}{ Alta Aplicación } & \multicolumn{3}{|c|}{ Alta Aplicación } & \multicolumn{2}{|c|}{ Alta Aplicación } & \multicolumn{2}{|c|}{ Moderada Aplicación } \\
\hline \multicolumn{2}{|c|}{ Media de la dimensión } & \multicolumn{9}{|c|}{3,54} \\
\hline \multicolumn{2}{|c|}{ Categoría de la dimensión } & \multicolumn{9}{|c|}{ Alta Aplicación } \\
\hline
\end{tabular}


En la Tabla anterior se destacan las técnicas de la PNL utilizadas por los líderes de empresas, que el indicador calibración arrojó que un 36\% de la población encuestada siempre determina, a través de su respiración, cuándo un colaborador se encuentra molesto, analiza las acciones de cada uno de sus colaboradores de proyecto, visualiza sus gestos y observa con detalle el comportamiento de sus colaboradores, involucrados e interesados del proyecto. Mientras tanto, un $28 \%$ de los encuestados respondieron con la alternativa de respuesta: algunas veces, un $16 \%$ con la opción casi siempre, un $12 \%$ la alternativa casi nunca y un $8 \%$ nunca.

El indicador dio una media de 3,53 ubicándose según el baremo de interpretación de los promedios en una alta aplicación, el cual, una vez comparado con la media de la dimensión que fue 3,54; se encuentra ligeramente por debajo de su nivel, lo cual indica que los líderes de proyectos manejan las técnicas de calibración, desconocen a profundidad los beneficios potenciales que pueden maximizar su gestión de las comunicaciones en los proyectos, ya que los líderes que desarrollan la calibración pueden establecer una relación directa con su interlocutor, al crear a través de la sincronización, de los gestos y forma de hablar, un buen clima de confianza entre las partes.

Los resultados que se obtuvieron difieren del argumento de Sambrano (2005), donde hace referencia a que la calibración significa aprender a conocer en forma precisa, el estado mental de las personas, es una habilidad que todos tenemos y empleamos en nuestra vida cotidiana, cuando se desarrolla y se optimiza, se logra la excelencia en la comunicación.

Asimismo, en la Tabla 2, se muestra que, para el indicador anclaje, el 36\% de la población encuestada, identificaron que siempre controlan sus emociones ante situaciones conflictivas, cuentan algunas vivencias para sintetizar situaciones específicas con sus colaboradores, cuando desean manifestar algo lo hacen a través del uso de representaciones gráficas y cuando se dirigen a su equipo de trabajo, manifiestan opiniones a través de emociones. Mientras que, un $28 \%$ respondieron casi siempre, un $20 \%$ casi nunca, un $12 \%$ algunas veces y un $4 \%$ nunca.

El indicador anclaje arrojó una media de 3,50 ubicándose en una categoría de alta aplicación que comparado con el resultado de la media de la dimensión de 3,54 se encuentra por debajo de la misma; esto permitió inferir que, los líderes de proyectos de las empresas mixtas del sector petrolero del estado Zulia, requieren anclar emociones para cambiar situaciones donde la comunicación ha sido cortada, donde existan paradigmas o barreras para expresar sus ideas o pensamientos. Por tanto, todas las personas evocan recuerdos a partir de algún momento en particular revivido, es entonces, a partir de esos recuerdos positivos donde el líder debe producir el anclaje, en sus colaboradores, interesados, involucrados, entre otros.

Los resultados obtenidos difieren de la opinión de Mohl (2006), quien manifiesta que establecer un estímulo externo y ligarlo deliberadamente con la experiencia, con el objeto de resucitarla en el momento deseado, en términos de programación neurolingüística es conocido como anclaje. 
Del mismo modo, en la tabla 2, se muestra que, para el indicador rapport, la población encuestada respondió que un 44\% siempre, aceptan a su colaborador tal cual es, mantienen interés en mejorar la calidad de la comunicación con sus colaboradores, muestran receptividad al momento de escuchar el planteamiento de sus colaboradores y fomentan un ambiente empático con sus colaboradores. Mientras que, un $28 \%$ respondió casi siempre, un $16 \%$ algunas veces, un $8 \%$ nunca y un $4 \%$ casi nunca.

El indicador arrojó una media de 3,75 que lo ubicó en una categoría de alta aplicación y le colocó por encima de la media de la dimensión, la cual es de 3,54; con el resultado mostrado se evidenció que, los líderes de proyectos utilizan la técnica para sintonizar con su equipo de proyecto, pero se hace necesario conocer a fondo el beneficio y la forma de crear alianzas estratégicas con el desarrollo de la misma.

Lo anterior, fue sustentado por la opinión de Mohl (2006), quien concibe al rapport como una forma de crear sintonía con las personas, donde existe un contacto directo, caracterizado por respeto, confianza y aceptación mutua. La calidad de la misma, dependerá de la actitud del individuo, de su capacidad para aceptar al otro, siendo además necesario, que se sienta receptivo para poder dedicarse plenamente a su interlocutor.

Por otra parte, para el indicador reencuadre, se muestra en la tabla 2 , que, el $28 \%$ de los líderes de proyectos manifestaron que siempre, toman en cuenta las expectativas de sus colaboradores en el proyecto al momento de tomar una decisión, manifiestan interés en determinar el estado emocional de sus colaboradores, evocan recuerdos positivos para cambiar situaciones emocionales y toman aprendizaje a partir de conductas manifestadas por sus colaboradores como respuesta a situaciones específicas. Mientras que, un $24 \%$ de los encuestados respondieron casi siempre, un $20 \%$ algunas veces, un $20 \%$ casi nunca y un $8 \%$ nunca.

La media del indicador fue de 3,37 ubicándose en una categoría de moderada aplicación la cual, al ser comparada con la media de la dimensión de 3,54, se encuentra considerablemente por debajo, por lo que se hizo necesario hacer énfasis en el desarrollo de esta técnica para el manejo de situaciones conflictivas y problemas comunicacionales. Los líderes que ejecuten esta técnica conseguirán resultados positivos, puesto que lograrán traer a su lado a quienes de momento se muestren resistentes a cambios; además, la técnica ofrece crear vínculos poderosos que permiten cambiar el estado emocional, también funciona como una técnica para la persuasión y la atracción.

Los resultados anteriores difirieron con lo expresado por los autores O'Connor y Seymour (2000), quienes infieren que el significado de cualquier evento cambia, cuando se cambia el marco, por lo cual también cambia las respuestas y el comportamiento.

De igual manera, es importante mencionar el resultado obtenido por la dimensión, la cual tuvo una media de 3,54 categorizada según baremo de medición en una alta aplicación, por parte de los líderes de proyectos, en la utilización de las técnicas de la PNL no obstante, se encontraron debilidades que serán expuestas más adelante.

Ahora bien, lo anteriormente planteado es soportado por Garay (2012), quien se refiere a las técnicas como aquellas que permiten desarrollar procesos de comunicación y cambio 
con excelencia, promoviendo flexibilidad del comportamiento, el pensamiento estratégico y la comprensión de los procesos mentales; éstas pueden ser aplicadas tanto a situaciones personales, grupales y organizacionales por ser un modelo basado en interacciones humanas.

\section{CONCLUSIONES}

$\mathrm{P}$ or otra parte, en función de los resultados obtenidos relativos a la dimensión técnicas de la PNL utilizadas por los líderes de empresas, se puede concluir que, aunque los líderes hacen uso de las técnicas del PNL, pudieran obtener un mayor beneficio si profundizan en su conocimiento.

El manejo de las técnicas de PNL, especialmente la técnica del reencuadre, por cuanto al cambiar el contexto emocional, las personas perciben un significado diferente de las experiencias vividas, lo cual conlleva a resultados fisiológicos positivos que impactan la conducta y generan cambios, por lo tanto estas herramientas son relevantes para el logro de un proceso de liderazgo efectivo en los líderes de las empresas petroleras.

\section{REFERENCIAS}

Aprender PNL. (2013). [Página web en línea] (http://aprenderpnl.com/tecnicas-pnl/) (consulta: 2015, febrero 10).

Arocha. (2005). PNL organizacional. Editorial Júpiter, 2da. Edición, Caracas, Venezuela

Astorga, F. (2013). PNL y éxito [Página web en línea]. Extraído de http://pnlyexito.com/ blog/tecnicas-de-pnl/) (consulta: 2015, febrero 10)

Bandler, R., y Grinder, J (2013). De sapos a príncipes. Editorial Cuatro Vientos, Santiago de Chile, Chile

Carpio, M., y Flores, C (2007). Cambio y transformación. Manual de Desarrollo Personal. Grupo Gráfico 5 C.A., Caracas, Venezuela

Carpio, M., y Perera, N. (2009). ¡Mira! ¡Escucha! Y ¡Conéctate! Con la PNL. Talitip, s.r.l., Caracas Venezuela

Carrión, L (2005). Inteligencia emocional con PNL. Editorial Edaf S.A., Madrid España
Garay, R. (2012). [Página web en línea] (http:// sceu.frba.utn.edu.ar/course/herramientasde-pnl-programacion-neurolinguisticaintegrando-el-modelo-de-cerebrointegral/) (consulta: 2015, diciembre 10)

García, L. (2004). Las empresas venezolanas. Editorial Norma, Caracas, Venezuela

Gordillo, A. (2005). El Parasistema JurídicoAdministrativo. Caracas, Venezuela. Jurídica venezolana

Mohl, A (2006). Aprendiz de Brujo II. Editorial Sirio, S.A., Málaga España

O'Connor, J., y Seymour, J. (2000). PNL para formadores. Ediciones Urano, Barcelona, España

Sambrano, J. (2005). PNL, para todos, El modelo de la excelencia. Caracas, Venezuela. Grupo Editorial ALFA 